\title{
Nuevos anticoagulantes orales: actualización
}

\author{
Alejandro Berkovits ${ }^{1}$, Diego Mezzano ${ }^{2}$. \\ 1 Departamento de Hemato-Oncología, Clínica Alemana de Santiago. \\ 2 Departamento Hematología-Oncología, Escuela de Medicina, Pontificia Universidad Católica de Chile. \\ Los autores no declaran conflictos de interés relacionados con el tema de este manuscrito.
}

Fuente de Financiación: Parcialmente por Proyecto FONDECYT 113853.

\section{Resumen:}

Los anticoagulantes orales clásicos del tipo cumarinas han estado disponibles para uso clínico por más de medio siglo. Tienen gran eficacia para tratar o prevenir trombosis y tromboembolias, y son drogas cuyo uso ha aumentado con el mejor conocimiento clínico, el aumento de los factores de riesgo y el envejecimiento de la población. Entre sus desventajas se incluyen la alta variabilidad de su efecto en cada sujeto y entre individuos, la influencia del nivel de ingesta de vitamina $\mathrm{K}$, la necesidad de control periódico del nivel de anticoagulación, su interacción con múltiples drogas. Si bien, el rango terapéutico está estandarizado, es estrecho, haciendo que el tiempo en rango terapéutico sea de $\approx 60 \%$.
Por estas limitaciones, se han creado nuevos anticoagulantes orales (NACOs), siendo progresivamente aprobados para uso clínico por agencias internacionales. Genéricamente, son de 2 tipos: inhibidores selectivos de trombina (dabigatrán) o de FXa (rivaroxabán, apixabán, edoxabán y betrixabán). Los NACOs se caracterizan por su dosificación una o dos veces al día, rapidez de acción, corta vida media en la circulación, predictibilidad de su efecto, dosis preestablecidas, sin necesidad de control periódico y con escasa o nula interacción con otras drogas. Estas ventajas no se han traducido en la mayoría de los ensayos en un superior efecto antitrombótico o menor riesgo de sangrado, y en su mayoría (salvo dabigatrán) carecen de antídoto específico demostrado.

\section{Correspondencia:}

Dr. Diego Mezzano

Laboratorio de Trombosis y Hemostasia

Escuela de Medicina, P. Univ. Católica de Chile.

Teléfono: +56 223543774

dmezzano@med.puc.cl 


\section{New oral anticoagulants: a review}

Vitamin K inhibitors, coumarins, have been used for more than 50 years with no dispute by other drugs. Coumarins have demonstrated great efficacy in the treatment and prophylaxis of thrombotic and thromboembolic disorders, and their use has increased progressively with the advance of clinical knowledge as well as the increase of risk factors and aging of the population. Limitations of coumarins include great variability intra- and inter-individuals, the influence of foods rich in vitamin $\mathrm{K}$, the need for periodical assessment of the anticoagulant level and drug interactions. The therapeutic range is standardized using the INR (International Normalized Ratio). However, the therapeutic window is narrow, with frequent periods of either over or under-dosing, with the concomitant increase of bleeding and thrombotic risks, respectively. Long-term accredited anticoagulant clinics and clinical trials report that, at best, only $\approx 60 \%$ of time in treatment the patients are within the therapeutic range.
These limitations have created the need for new oral anticoagulants (NOACs), and several of them have been approved for clinical use by international agencies after exhaustive and specific clinical trials. Generically, NACOs are belong in two types: selective inhibitors of thrombin (dabigatran) or FXa (rivaroxaban, apixaban, edoxaban and betrixaban). NOACs are prescribed once or twice daily, the onset of action is very fast, have a low $\mathrm{T} 1 / 2$ in the circulation, their effects are highly predictable, doses are pre-established, do not need laboratory control and have a low rate of interaction with other drugs. Despite these advantages most clinical trials have shown NOACs to be not inferior with respect to coumarin. However, NOACs have no clear advantages over warfarin in antithrombotic effect or bleeding reduction. Furthermore, most of them (except dabigatran) have no specific antidotes yet.

Keywords: New Anticoagulants, Rivaroxaban, Apixaban, Dabigatran, Betrixaban, Apixaban. 


\section{Introducción:}

La anticoagulación oral con inhibidores de vitamina K (cumarinas) sigue dominando en nuestro país el tratamiento y prevención de trombosis venosa profunda (TVP) y enfermedad trombo-embólica (ETE). La efectividad de las cumarinas no ha sido superada aún por los nuevos anticoagulantes orales (NACOs), pero estos tienen importantes limitaciones, incluyendo su estrecho rango terapéutico y gran variabilidad inter- e intra-individual. Requieren control periódico con INR (International Normalized Ratio), un tiempo de protrombina corregido que homogeniza los resultados obtenidos entre laboratorios que utilizan distintos equipos y reactivos. Se usa mayoritariamente un rango de INR de 2-3: bajo este umbral, el paciente no está suficientemente protegido de trombosis y sobre él está expuesto a sangrado patológico. Éste puede alcanzar 30\% al año con INR $>51$. Nuevos anticoagulantes orales (NACOs) han emergido y ganado lugar en la práctica clínica. Los NACOs tienen farmacocinética y farmacodinamia más predecible, mayor rango terapéutico y dosis fija sin necesidad de monitorización. En la actualidad se usan en clínica 2 tipos de NACOs: inhibidores directos del FX activado (FXa) o inhibidores de la trombina FIIa), ambas enzimas claves en la vía final de la coagulación. El incremento exponencial del uso de NACOs nos motiva a actualizar nuestra revisión de $2011 .{ }^{2}$

\section{Esquema de la hemostasia y sitios de acción de los anticoagulantes.}

La ruptura vascular, el daño endotelial o los accidentes de placa ateromatosa exponen factor von Willebrand, colágeno y otras proteínas adhesivas, que inician la adhesión y posterior activación (agregación-secreción) plaquetarias. El Factor Tisular (FT) presente en células subendoteliales gatilla el inicio de la coagulación. La membrana de las plaquetas activadas provee una superficie rica en fosfolípidos aniónicos donde se ensamblan los complejos macromoleculares y las reacciones enzimáticas que concluyen en la generación de FXa, trombina y formación del coágulo de fibrina. ${ }^{3}$ El desarrollo del proceso de la coagulación sobre membranas celulares es la base del modelo actual de Hemostasia Basada en Células ${ }^{4,5,6,7,8}$. Por razones de simplicidad, el antiguo esquema clásico de activación en cascada de los factores de la coagulación se muestra en la Figura 1. Se apuntan los sitios de acción de los anticoagulantes clásicos (cumarinas y heparinas) y, destacados los sitios de inhibición de los NACOs. Éstos están reemplazando

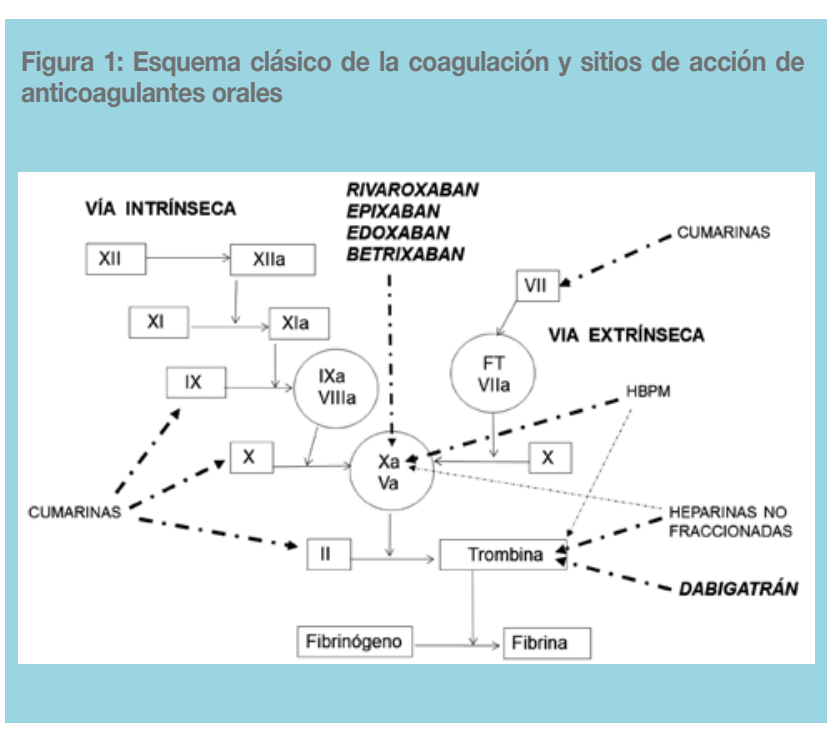

a los anticoagulantes clásicos, en tratamiento y prevención de TVP y ETE, la principal causa de morbimortalidad de pacientes hospitalizados y la segunda causa de muerte en pacientes con cáncer. ${ }^{9,} 10$

\section{Anticoagulantes tradicionales: Antagonistas de la vitamina $\mathrm{K}$.}

Los anticoagulantes orales cumaríninicos más usadas son la warfarina ( $\mathrm{T}^{1 / 2}$ de 35 a 45 horas) y en Chile el acenocumarol ( $\mathrm{T}^{1 / 2}$ de 8 a 24 horas). Esta depuración más rápida del acenocumarol es útil en caso de sobredosis, pero se asocia a mayor inestabilidad del rango terapéutico. ${ }^{11}$ Las cumarinas interfieren la reducción de la vitamina $\mathrm{K}$ de su forma epóxido oxidada a hidroquinona, su forma activa. La vitamina $\mathrm{K}$ es cofactor en la $\gamma$-carboxilación post-ribosomal de ácidos glutámicos en los FII, FVII, FIX y FX. E defecto de carboxilación bloquea la unión de estos factores a fosfolípidos aniónicos en membranas celulares (especialmente plaquetas) y el ensamblaje de complejos macromoleculares necesarios para la secuencia enzimática de la coagulación. ${ }^{12,13,14,11}$

Las cumarinas se usaron sin competencia por más de medio siglo, tienen buena absorción y bajo costo. En sobredosis, su efecto puede revertirse utilizando principalmente concentrados liofilizados de complejos protrombínicos. Sus principales desventajas son las múltiples interacciones con fármacos y alimentos que modifican su metabolismo generando variabilidad en un mismo paciente e inestabilidad dosis-respuesta. Por ello, debe controlarse periódicamente y ajustar el INR en rango terapéutico. 


\begin{tabular}{|c|c|c|c|c|c|}
\hline & Dabigatrán & Rivaroxabán & Apixabán & Edoxabán & Betrixabán \\
\hline Mecanismo & $\begin{array}{l}\text { Inhibidor } \\
\text { trombina }\end{array}$ & Inhibidor Xa & $\begin{array}{l}\text { Inhibidor } \\
\text { Xa }\end{array}$ & $\begin{array}{l}\text { Inhibidor } \\
\text { Xa }\end{array}$ & $\begin{array}{l}\text { Inhibidor } \\
\mathrm{Xa}\end{array}$ \\
\hline $\begin{array}{l}\text { Nombre } \\
\text { Comercial. }\end{array}$ & Pradaxa ${ }^{\circledR}$ & Xarelto® & Eliquis® & Savaysa ${ }^{\circledR}$ & \\
\hline $\begin{array}{l}\text { Concentración } \\
\text { máxima }\end{array}$ & $1.5-3 h$ & $2-4 h$ & $1-3 h$ & $1-1.5 \mathrm{~h} \quad 1$ & \\
\hline Vida media & $2-17 h$ & $9-13 h$ & $8-15 h$ & $9-11 h$ & $19 \mathrm{~h}$ \\
\hline Excreción renal & $80 \%$ & $66 \%$ & $25 \%$ & $35 \%$ & $5 \%$ \\
\hline Dosis prevención & $150-220 \mathrm{mg} / \mathrm{d}$ & $\begin{array}{c}10 \mathrm{mg} / \mathrm{d} \\
150 \mathrm{mg} / 12 \mathrm{~h}\end{array}$ & $2.5 \mathrm{mg} / 12 \mathrm{hs}$ & $(-)$ & $80 \mathrm{mg} / \mathrm{d}$ \\
\hline Dosis tratamiento & $15 \mathrm{mg} / 12 \mathrm{hs}$ & $\begin{array}{l}\text { x21d. Luego: } \\
20 \mathrm{mg} / \mathrm{d}\end{array}$ & $5 \mathrm{mg} / 12 \mathrm{hs}$ & $60 \mathrm{mg} / \mathrm{d}$ & $(-)$ \\
\hline Dosis de inicio & $\begin{array}{l}\text { Puente } \\
\text { HBPM }\end{array}$ & Desde inicio & Desde inicio & $\begin{array}{l}\text { HBPM } 5 \text { d previos } \\
\text { sin puente }\end{array}$ & \\
\hline Reversión & $\begin{array}{l}\text { Idarucizumab } \\
\text { (50) }\end{array}$ & Andexanet & $\rightarrow$ Fase $3(51)$ & & \\
\hline
\end{tabular}

\section{Nuevos anticoagulantes orales (Tabla 1)}

\section{Inhibidores de la trombina}

Dabigatrán: antagoniza competitivamente la trombina, inhibiendo tanto su fracción soluble como la unida a fibrina. Esta última propiedad lo diferencia de las heparinas. Se usa en dosis fija, sin monitorización de su efecto, y debe administrarse 2 veces/día (BID). Al igual que los otros NACOs, están contraindicados en embarazo y lactancia. Recientemente se aprobó un antídoto para su sobredosis, un anticuerpo monoclonal anti-Dabigatrán (idarucizumab). ${ }^{14-17}$

La prodroga, Dabigatrán etexilate, se metaboliza en el hígado a Dabigatrán (forma activa). Su biodisponibilidad es 7.2\%, con inicio de acción de 1.5-3 horas con T1/2 de 12-17hs. Su excreción es mayoritariamente renal (80\%), y por ello, está contraindicado en pacientes con clearance de creatinina $<30 \mathrm{ml} / \mathrm{min} .{ }^{18}, 19$

\section{Inhibidores de FXa}

Actúan en una etapa previa en la cascada de la coagulación. Inhiben al FXa, parte del complejo protrombinasa (FXa-FV) y al FXa asociado a trombina. Al igual que los inhibidores de la trombina, no requieren monitorización de niveles de anticoagulación y se administran en dosis fija. Hasta la fecha carecen de antídoto específico demostrado.

- Rivaroxabán: Tiene $80 \%$ de biodisponibilidad, rápido inicio de actividad, con máximo plasmático en 2 a 4 horas. Su T1/2 varía según la función renal, promediando de 5-9 hs en los jóvenes y 11-13 hs en adultos mayores, con clearance de creatinina $<50 \mathrm{ml} / \mathrm{min}$. La excreción es $66 \%$ renal (36\% como droga y $30 \%$ como metabolito). El resto (28\%) se elimina por deposiciones. Tiene baja interacción con otros fármacos, pero los azoles e inhibidores de proteasa-VIH pueden aumentar su nivel plasmático. ${ }^{20}$

- Apixabán: Tiene biodisponibilidad >50\%, su máximo plasmático y $\mathrm{T}^{1 / 2}$ son de 3 y 12 horas, respectivamente. La metabolización es principalmente hepática, sin inducción o inhibición a nivel de citocromos y, por tanto, su interacción con otras drogas es mínima. La excreción es mayoritariamente fecal, siendo sólo $25 \%$ por vía renal. ${ }^{21}$ - Edoxabán: Su máximo plasmático es en 1-2 horas, y acción máxima anti-FXa en $1.5 \mathrm{hs}$. Su T1/2 y biodisponibilidad son de 10-14 horas y $62 \%$, respectivamente. La unión a proteínas plasmáticas es $55 \%$, menor que apixabán (87\%) y rivaroxabán (90\%). Su eliminación es $35 \%$ por vía renal. ${ }^{22}$

- Betrixabán: Es el quinto NACO aprobado por la F.D.A., con características farmacocinéticas únicas, como escasa eliminación renal y T1/2 de $19 \mathrm{hs}$. Se administra una vez al día, con baja interacción de drogas. ${ }^{23}$ La aprobación de cada NACO para uso clínico ha seguido pautas bastante estereotipadas, similares a las usadas en la aprobación de las heparinas de bajo peso molecular en los años 90. En general, se ha probado su capacidad profiláctica, y en la mayoría en el tratamiento de TVP/ETE en pacientes con cirugía traumatológica de reemplazo de cadera y/o rodilla, en embolia arterial secundaria a fibrilación auricular no valvular. Se ha incorporado también su uso en la prevención de TVP/ 
ETE en pacientes no quirúrgicos postrados en cama por tiempos prolongados.

\section{Evidencias clínicas de NACOs en prevención de tromboembolismo arterial en pacientes con fibrila- ción auricular no valvular (FANV). (Tabla 2)}

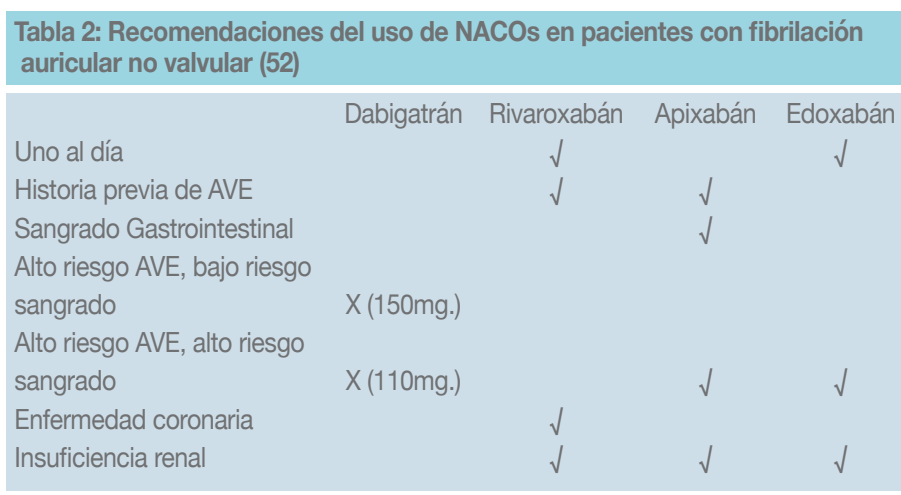

\section{Dabigatrán}

El estudio RE-LY evaluó >18.000 individuos con FANV. Se comparó dos dosis de Dabigatrán, 110mg BID y $150 \mathrm{mg}$ BID con warfarina en prevención secundaria de fenómenos embólicos cerebrales y sistémicos. Se excluyó pacientes con insuficiencia renal (clearance de creatinina $<30 \mathrm{ml} / \mathrm{min}$ ). A dos años las tasas anuales de embolias fueron $1.53 \%$ con $110 \mathrm{mg}$ BID y $1.69 \%$ con warfarina (RR de 0,91 (95\% IC $0,74-$ 1,11), demostrando no-inferioridad. Con $150 \mathrm{mg}$ BID la incidencia de embolias fue de $1,11 \%$ que comparado con warfarina muestra una reducción del RR de $44 \%$ (RR 0,66 (95\% IC 0,53-0.82)), siendo significativamente superior a warfarina. La mortalidad anual fue de $3.7 \%$ y $3.6 \%$ con dosis bajas y altas de Dabigatrán, respectivamente, y $4.13 \%$ con warfarina, diferencia no significativa. Dabigatrán 110mg BID demostró menos sangrados que $150 \mathrm{mg}$ BID y que warfarina. Un análisis de subgrupo mostró que en pacientes con AVE transitorio (TIA) o AVE previo, ambas dosis de Dabigatrán disminuyeron en forma significativa los episodios de accidente cerebrovascular hemorrágico. Si bien este estudio fue diseñado para mostrar "no inferioridad", éste es el primer trabajo publicado que demuestra que un anticoagulante oral supera a warfarina en la prevención de eventos tromboembólicos en FANV usando dosis altas, sin aumento significativo en los efectos adversos. ${ }^{24}$ El año 2016 se publicó un análisis de subgrupo del estudio Re-LY incluyendo 3950 pacientes con enfermedad valvular, mostrando similares resultados en pacientes con FANV. ${ }^{25}$

\section{Rivaroxabán}

El estudio ROCKET-AF incluyó $>14.000$ pacientes con FANV y alto riesgo de embolía (C.H.A.D.S. score $>2$ en 90\%) asignados a $20 \mathrm{mg}$ de rivaroxabán (15 mg en hipofunción renal) o warfarina. Se demostró no inferioridad a la warfarina en el criterio principal de valoración combinado, que incluyó AVE y embolia sistémica. Las tasas de hemorragia fueron similares en ambos grupos, con menos episodios de sangrados intracerebrales y mortales en el grupo de rivaroxaban. ${ }^{26}$

\section{Apixabán}

El estudio ARISTOTLE, con $>18.000$ pacientes con FANV mostró que apixabán en dosis de 5mg BID fue superior a warfarina en prevención de AVE y embolias sistémicas, con reducción RR de 21\% (RR 0.79 ,95\% CI $0.66-0.95 ; \mathrm{p}<0.0001$ para no inferioridad y $\mathrm{p}=0.01$ para superioridad) y disminución significativa de la tasa de hemorragia intracerebral $(0.24 \%$ y $0.47 \%$ al año con apixabán y warfarina, respectivamente) mayormente en pacientes con AVEs previos; se observó además menor tendencia a mortalidad global.

\section{Edoxabán}

Fue evaluado en $>20.000$ pacientes con FANV y alto riesgo embólico en dosis de $30 \mathrm{mg}$ y $60 \mathrm{mg}$ versus warfarina, para la disminución del riesgo de embolias cerebrales y sistémicas. La tasa de eventos primarios fue de $1.8 \%$ con warfarina comparado con $1.57 \%$ y $2.04 \%$ con dosis bajas y altas, respectivamente, con significancia estadística para no inferioridad. Las tasas de eventos adversos, principalmente sangrado o muerte fueron menores con ambas dosis de edoxaban. ${ }^{27-31}$

\section{Evidencias clínicas para el tratamiento de TVP/ ETE (Tabla 3)}

\section{Dabigatrán}

Los estudios RE-COVER I y II compararon tratamiento de TVP/ tromboembolismo pulmonar (TEP) en pacientes

\begin{tabular}{|c|c|c|c|}
\hline \multicolumn{4}{|c|}{$\begin{array}{l}\text { Tabla 3: Efectividad de NACOs en prevención de recurrencia de } \\
\text { enfermedad tromboembólica venosa y muerte en pacientes } \\
\text { con TVP/TEP }\end{array}$} \\
\hline & NACOs n/N (\%) & Warfarina n/N (\%) & RRA \% $(95 \% \mathrm{Cl})$ \\
\hline Dabigatrán & 60/2553 (2.7) & $55 / 2554$ (2.4) & $0.3(-0.6,1.0)$ \\
\hline Rivaroxabán & $86 / 4130(2.1)$ & $95 / 4131$ (2.3) & $-0.2(-0.8,0.4)$ \\
\hline Apixabán & $59 / 2609$ (2.3) & $71 / 2653(2.7)$ & $-0.4(-1.3,0.4)$ \\
\hline Edoxabán & 130/4118 (3.2) & $146 / 4122(3.5)$ & $-0.4(-1.2,0.4)$ \\
\hline
\end{tabular}


con Dabigatrán 150mg BID o warfarina durante 6 meses. Se demostró similar incidencia de episodios de TVP recurrente o muerte relacionada en ambos grupos. La frecuencia de sangrado mayor fue similar, pero la frecuencia total de eventos fue significativamente menor en el grupo con Dabigatrán y un análisis suplementario mostró que el único grupo potencialmente beneficiado con warfarina es aquel con TVP/TEP previos. ${ }^{32,33}$

La evaluación de la extensión del tratamiento, comparado con warfarina (RE-MEDY) o placebo (RE-SONATE) mostró que el uso extendido de tratamiento anticoagulante (mediana de 480 días) disminuyó en $92 \%$ la recurrencia o muerte relacionada a TVP/TEP, pero con aumento del sangrado clínicamente relevante. ${ }^{34}$

\section{Rivaroxabán}

Los estudios EINSTEIN-DVT y EINSTEIN-PE compararon la eficacia y seguridad de rivaroxabán (15mg BID por 21 días y después $20 \mathrm{mg} /$ día por 21 días) vs tratamiento estándar (enoxaparina $1 \mathrm{mg} / \mathrm{kg}$ BID y luego warfarina o acenocumarol) en 3449 pacientes con episodio de TVP sin TEP demostrado y 4832 con TEP, respectivamente. La edad media fue de 58 años, con $65 \%$ de episodios no provocados y sin insuficiencia renal. El I.N.R. estuvo en rango terapéutico aproximadamente $60 \%$ del tiempo, durante los 3, 6 o 12 meses de tratamiento, respetando la indicación del médico tratante. Los objetivos principales fueron recurrencia de TVP y muerte asociada a ella. Los resultados del EINSTEIN-DVT mostraron incidencia de objetivo primario en $2.1 \%$ para Dabigatrán y $3 \%$ para warfarina, con significancia estadística para no inferioridad $(95 \%$ CI, 0,11-1,04; $\mathrm{p}<0,001)$, y sin diferencias en tasas de sangrado. El estudio EINSTEIN-PE mostró similares resultados con disminución absoluta de eventos primarios en $0.5 \%$, cifra no inferior a warfarina $(\mathrm{p}<0.003)$, y con menos sangrado mayor ${ }^{34,35}$.

Finalizado el tratamiento anticoagulante se evaluó extender la profilaxis secundaria con rivaroxabán a 20mg/ día comparado con placebo por 6-12 meses (EINSTEIN-EXT). Rivaroxabán mostró una reducción del RR del objetivo primario en $88 \%$, pero con aumento moderado de sangrado. ${ }^{36}$

\section{Apixabán}

La comparación entre apixabán y tratamiento estándar (puente enoxaparina-warfarina) (estudio AMPLIFY) se realizó en $>5.000$ pacientes. Apixabán con carga de $10 \mathrm{mg}$ BID por 7 días y luego $5 \mathrm{mg}$ BID fue al menos no inferior al tratamiento estándar. El mayor hallazgo fue una importante reducción en sangrados mayores con apixabán (RRR de 69\%). ${ }^{37}$ Extendiendo a 12 meses o más el tratamiento (estudio AMPLIFY), apixabán redujo el riesgo absoluto de recurrencia de TVP/TEP o mortalidad en $7.8 \%$ y $7.4 \%$ con dosis de 2.5 y $5 \mathrm{mg}$, respectivamente, comparado con placebo. No hubo diferencias en sangrados. ${ }^{38}$

\section{Edoxabán}

El estudio Hosukai-VTE demostró que edoxabán fue al menos no inferior $(\mathrm{p}<0.001)$ a warfarina en evitar recurrencias en pacientes con TVP aisladas y TEP, y reduciendo en $19 \%$ el sangrado mayor. Los pacientes con edoxabán y warfarina recibieron puente inicial con heparina por al menos 5 días. La dosis de edoxabán fue de $60 \mathrm{mg} /$ día en pacientes con clearance de creatinina $>50 \mathrm{ml} / \mathrm{min}$ y $30 \mathrm{mg} /$ día en aquellos entre 30 y $50 \mathrm{ml} /$ min, o con peso corporal $<60 \mathrm{Kg}$. ${ }^{39}$

\section{Tromboprofilaxis en pacientes ortopédicos con alto riesgo de TVP/TEP}

\section{Dabigatrán}

Los estudios RE-NOVATE I y II, compararon Dabigatrán (150mg y $220 \mathrm{mg} /$ día en una sola toma) con enoxaparina (40mg/día por 28 a 35 días) en pacientes sometidos a reemplazo total de cadera. No hubo diferencias significativas en incidencia de eventos tromboembólicos y mortalidad ni en episodios de sangrado.40 El Estudio RE-MODEL comparó Dabigatrán en dos dosis (150mg y $220 \mathrm{mg} /$ día) con enoxaparina (40mg/día) en profilaxis de artroplastia de rodilla. El resultado en artroplastía de cadera fue similar al anterior. ${ }^{41}$

\section{Rivaroxabán}

El estudio RECORD 1 evaluó la efectividad de rivaroxabán en la prevención de la enfermedad tromboembólica en pacientes sometidos a artroplastía total de cadera. Se randomizó 4.541 pacientes para recibir rivaroxabán $10 \mathrm{mg} /$ día o tratamiento estándar (enoxaparina $40 \mathrm{mg} /$ día) hasta $35 \pm 4$ días postcirugía. Se observó reducción absoluta (RRA) de episodios de TVP/ETE o muerte en 2,6\% con el inhibidor de FXa en comparación con tratamiento estándar (-2.6\%, CI 95\% (-3.7\% a $1.5 \%) \mathrm{p}<0.001)$. No hubo diferencias en los episodios de sangrado. ${ }^{42}$

Resultados similares se observaron comparando rivaroxabán por $35 \pm 4$ días postcirugía y dosis acortada de 
enoxaparina (40mg/día por 12 días y luego placebo) en el mismo grupo de pacientes (RECORD 2). La reducción del RR de TVP/TEP o muerte fue de 79\%. ${ }^{43}$

El estudio RECORD 3 comparó rivaroxabán y enoxaparina (ambos en dosis preventivas) por $12 \pm 2$ días en 2531 pacientes sometidos a reemplazo total de rodilla. Se observó reducción significativa en episodios de trombosis venosa distal, pero sin diferencias en trombosis proximales, tromboembolismo pulmonar o mortalidad. ${ }^{44}$ El estudio RECORD 4 es análogo al RECORD 3, pero con enoxaparina $30 \mathrm{mg} \mathrm{c} / 12$ horas por 14 días. Rivaroxabán redujo en $3.2 \%$ el riesgo absoluto de TVP, TEP y muerte de cualquier causa, sin diferencias en la tasa de sangrados mayores. ${ }^{45}$

\section{Apixabán}

Se evaluó en tromboprofilaxis en cirugía de cadera o rodilla, siendo similar a enoxaparina en el objetivo primario de TVP sintomática, con RR de 0.82 (0.41-1.64) y menor sangrado clínicamente relevante (RR 0.82, 95\% CI 0.69-0.98; $\mathrm{p}=0.049) .46$

\section{Tromboprofilaxis en pacientes médicos de alto riesgo de TVP/TEP}

\begin{tabular}{|c|c|c|c|}
\hline & NACOs n/N (\%) & Warfarina $\mathrm{n} / \mathrm{N}(\%)$ & RRA \% $(95 \%$ Cl) \\
\hline Dabigatrán & $37 / 2553(1.4)$ & $51 / 2554(2.0)$ & $-0.5(-1.3,0.2)$ \\
\hline Rivaroxabán & $40 / 4130(1.0)$ & $72 / 4116(1.7)$ & $-0.8(-1.3,-0.3)$ \\
\hline Apixabán & $15 / 2676(0.6)$ & 49/2689 (1.8) & $-1.3(-1.8,-0.6)$ \\
\hline Edoxabán & $56 / 4118(1.4)$ & $66 / 4122(1.6)$ & $-0.2(0.8,-0.3)$ \\
\hline
\end{tabular}

\section{Rivaroxabán:}

El estudio MAGELLAN comparó dosis preventivas (10mg/día) utilizadas en protocolo extendido (35 días) y fue no inferior a enoxaparina $(40 \mathrm{mg} /$ día y utilizado $10 \pm 4$ días) en el objetivo primario (TVP asintomática proximal, sintomática proximal o distal, TEP no fatal o muertes relacionadas con enfermedad tromboembólica). Rivaroxabán, sin embargo, presentó mayor tasa de sangrado al día 10 y 35 post-inicio de tromboprofilaxis. ${ }^{47}$

\section{Apixabán:}

El estudio ADOPT comparó apixabán en dosis de $2.5 \mathrm{mg}$ BID por 30 días vs enoxaparina por 6 a 14 días, sin mostrar superioridad y con mayor tasa de sangrado. 48

\section{Betrixabán}

El estudio APEX comparó el beneficio de la tromboprofilaxis extendida con betrixabán $80 \mathrm{mg}$ por 35 días y enoxaparina por $10 \pm 4$ días en pacientes con dímero D elevado y/o más de 75 años (alto riesgo tromboembólico venoso). Betrixabán mostró ser superior a enoxaparina en la reducción del objetivo primario compuesto (similar a estudio MAGELLAN) sin aumento significativo en sagrados mayores. ${ }^{49}$

\section{Conclusiones:}

Los NACOs son una familia nueva de fármacos diseñados para reducir trombosis arterial y venosa. Comparados con los inhibidores de vitamina $\mathrm{K}$, tienen un rápido inicio de acción, mayor estabilidad, uso de dosis únicas y menor interacción con medicamentos, alimentos u otras sustancias. Se puede prescindir de controles periódicos, lo que en teoría aumenta la adherencia al tratamiento. El análisis de los diferentes estudios concluye que los 5 fármacos evaluados son al menos no inferiores al tratamiento estándar con similares perfiles de seguridad. En los pocos años de uso aprobado han ido reemplazando progresivamente a los inhibidores de vitamina K. Sin embargo, aún se debe tomar los resultados de estos grandes estudios clínicos con precaución, porque no siempre son extrapolables a poblaciones del "mundo real". Los NACOs tienen también algunas desventajas respecto a las cumarinas:

\section{Ausencia de método demostrado de monitorización:}

La ventaja que no requieran monitorización se convierte en problema si no existe método estandarizado ni evaluado de laboratorio que permita su rápida monitorización en situaciones de sangrado, procedimientos invasivos, cirugía de urgencia. Esta deficiencia ya se está corrigiendo con la aparición de pruebas que permiten determinar el nivel actual de anticoagulación.

Reversión de anticoagulación: Si bien los estudios están avanzando al respecto y Dabigatrán ya tiene un antídoto aprobado (idarucizumab), ${ }^{50}$ no existen aún para los fármacos inhibidores de FXa, pero se están diseñando moléculas para revertir su efecto. Así, están en curso los estudios fase 3 de andexanet como alternativa para su reversión del efecto anticoagulante. ${ }^{51}$ Los estudios que han utilizado los complejos protrombínicos para estos fármacos son heterogéneos y tienen el problema de la monitorización para verificar su reversión. 
Efectividad: Si bien los NACOs son al menos no inferiores a inhibidores de vitamina $\mathrm{K}$, el tiempo efectivo de anticoagulación con cumarinas es de sólo $60 \%$ del tiempo de uso. Esto apoya la gran efectividad de las cumarinas como anticoagulantes y plantea la duda respecto a si los NACOs, por su corta vida media, no anticoagulan eficientemente en las horas previas a las dosis diarias.
Situaciones especiales: La mayoría de los estudios excluyeron o incluyeron pequeñas poblaciones, con falla renal, obesidad, cáncer, trombofilias primarias o secundarias por lo que no siempre se puede extrapolar los resultados a todos los pacientes con TVP/ETE.

Costo: El costo actual del tratamiento con NACOs es muy alto con relación a las cumarinas, limitando su uso.

\section{Referencias}

1. STEHLE S, KIRCHHEINER J, LAZAR A, FUHR U. Pharmacogenetics of oral anticoagulants: a basis for dose individualization. Clin Pharmacokinet. 2008;47(9):565-94.

2. BERKOVITS A, AIZMAN A, ZUNIGA P, PEREIRA J, MEZZANO D. [New oral anticoagulant drugs]. Revista medica de Chile. 2011;139(10):1347-55.

3. WOOD JP, SILVEIRA JR, MAILLE NM, HAYNES LM, TRACY PB. Prothrombin activation on the activated platelet surface optimizes expression of procoagulant activity. Blood. 2011;117(5):1710-8.

4. HOFFMAN M, MONROE DM, 3rd. A cell-based model of hemostasis. Thromb Haemost. 2001;85(6):958-65.

5. HOFFMAN M, PAWLINSKI R. Hemostasis: old system, new players, new directions. Thromb Res. 2014;133 Suppl 1:S1-2.

6. PANES O, MATUS V, SAEZ CG, QUIROGA T, PEREIRA J, MEZZANO D. Human platelets synthesize and express functional tissue factor. Blood. 2007;109(12):5242-50.

7. HOFFMAN M, MONROE DM. Coagulation 2006: a modern view of hemostasis. Hematol Oncol Clin North Am.
2007;21(1):1-11.

8. KRETZ CA, VAEZZADEH N, GROSS PL. Tissue factor and thrombosis models. Arterioscler Thromb Vasc Biol. 2010;30(5):900-8.

9. SORENSEN HT, MELLEMKJAER L, OLSEN JH, BARON JA. Prognosis of cancers associated with venous thromboembolism. N Engl J Med. 2000;343(25):1846-50.

10. AGNELLI G, VERSO M, MANDALA M, GALLUS S, CIMMINIELLO C, APOLONE G, et al. A prospective study on survival in cancer patients with and without venous thromboembolism. Intern Emerg Med. 2014;9(5):559-67.

11. MANOLOPOULOS VG, RAGIA G, TAVRIDOU A. Pharmacogenetics of coumarinic oral anticoagulants. Pharmacogenomics. 2010;11(4):493-6.

12. WHITLON DS, SADOWSKI JA, SUTTIE JW. Mechanism of coumarin action: significance of vitamin $\mathrm{K}$ epoxide reductase inhibition. Biochemistry. 1978;17(8):1371-7.

13. WALLIN R, HUTSON SM. Warfarin and the vitamin K-dependent gamma-carboxylation system. Trends Mol Med. 
2004;10(7):299-302.

14. WAN H, YANG Y, ZHU J, WU S, ZHOU Z, HUANG B, et al. An in-vitro evaluation of direct thrombin inhibitor and factor $\mathrm{Xa}$ inhibitor on tissue factor-induced thrombin generation and platelet aggregation: a comparison of dabigatran and rivaroxaban. Blood coagulation \& fibrinolysis : an international journal in haemostasis and thrombosis. 2016;27(8):882-5.

15. POLLACK CV, JR., REILLY PA, BERNSTEIN R, DUBIEL R, EIKELBOOM J, GLUND S, et al. Design and rationale for RE-VERSE AD: A phase 3 study of idarucizumab, a specific reversal agent for dabigatran. Thrombosis and haemostasis. 2015;114(1):198-205.

16. BAGLIN T. Clinical use of new oral anticoagulant drugs: dabigatran and rivaroxaban. British journal of haematology. 2013;163(2):160-7.

17. AHRENS I, LIP GY, PETER K. New oral anticoagulant drugs in cardiovascular disease. Thrombosis and haemostasis. 2010;104(1):49-60.

18. EISERT WG, HAUEL N, STANGIER J, WIENEN W, CLEMENS A, VAN RYN J. Dabigatran: an oral novel potent reversible nonpeptide inhibitor of thrombin. Arteriosclerosis, thrombosis, and vascular biology. 2010;30(10):1885-9.

19. DUBOIS EA, COHEN AF. Dabigatran etexilate. British journal of clinical pharmacology. 2010;70(1):14-5.

20. MUECK W, STAMPFUSS J, KUBITZA D, BECKA M. Clinical pharmacokinetic and pharmacodynamic profile of rivaroxaban. Clin Pharmacokinet. 2014;53(1):1-16.

21. RAGHAVAN N, FROST CE, YU Z, HE K, ZHANG H, HUMPHREYS WG, et al. Apixaban metabolism and pharmacokinetics after oral administration to humans. Drug Metab Dispos. 2009;37(1):74-81.

22. BOUNAMEAUX H, CAMM AJ. Edoxaban: an update on the new oral direct factor Xa inhibitor. Drugs. 2014;74(11):1209-31.

23. CHAN NC, HIRSH J, GINSBERG JS, EIKELBOOM JW. Betrixaban (PRT054021): pharmacology, dose selection and clinical studies. Future Cardiol. 2014;10(1):43-52.

24. CONNOLLY SJ, EZEKOWITZ MD, YUSUF S, EIKELBOOM J, OLDGREN J, PAREKH A, et al. Dabigatran versus warfarin in patients with atrial fibrillation. N Engl J Med.
2009;361(12):1139-51

25. EZEKOWITZ MD, NAGARAKANTI R, NOACK $H$, BRUECKMANN M, LITHERLAND C, JACOBS M, et al. Comparison of Dabigatran and Warfarin in Patients With Atrial Fibrillation and Valvular Heart Disease: The RE-LY Trial (Randomized Evaluation of Long-Term Anticoagulant Therapy). Circulation. 2016;134(8):589-98.

26. PATEL MR, MAHAFFEY KW, GARG J, PAN G, SINGER DE, HACKE W, et al. Rivaroxaban versus warfarin in nonvalvular atrial fibrillation. N Engl J Med. 2011;365(10):883-91.

27. GRANGER CB, ALEXANDER JH, MCMURRAY JJ, LOPES RD, HYLEK EM, HANNA M, et al. Apixaban versus warfarin in patients with atrial fibrillation. N Engl J Med. 2011;365(11):981-92.

28. GIUGLIANO RP, RUFF CT, BRAUNWALD E, MURPHY SA, WIVIOTT SD, HALPERIN JL, et al. Edoxaban versus warfarin in patients with atrial fibrillation. $\mathrm{N}$ Engl J Med. 2013;369(22):2093-104.

29. GIUGLIANO RP. Once-daily edoxaban: a safer option than well-managed warfarin for patients with atrial fibrillation? Eur Heart J. 2014;35(28):1826-7.

30. GIUGLIANO RP, RUFF CT, ROST NS, SILVERMAN S, WIVIOTT SD, LOWE C, et al. Cerebrovascular events in 21105 patients with atrial fibrillation randomized to edoxaban versus warfarin: Effective Anticoagulation with Factor Xa Next Generation in Atrial Fibrillation-Thrombolysis in Myocardial Infarction 48. Stroke. 2014;45(8):2372-8.

31. GIUGLIANO RP, RUFF CT, WIVIOTT SD, NORDIO F, MURPHY SA, KAPPELHOF JA, et al. Mortality in Patients with Atrial Fibrillation Randomized to Edoxaban or Warfarin: Insights from the ENGAGE AF-TIMI 48 Trial. Am J Med. 2016;129(8):850-7 e2.

32. GOLDHABER SZ, SCHELLONG S, KAKKAR A, ERIKSSON H, FEURING M, KREUZER J, et al. Treatment of acute pulmonary embolism with dabigatran versus warfarin. A pooled analysis of data from RE-COVER and RE-COVER II. Thrombosis and haemostasis. 2016;116(4):714-21.

33. SCHULMAN S, KEARON C, KAKKAR AK, SCHELLONG $S$, ERIKSSON H, BAANSTRA D, et al. Extended use of dabigatran, warfarin, or placebo in venous thromboembolism. The New England journal of medicine. 2013;368(8):709-18. 
34. BAUERSACHS R, BERKOWITZ SD, BRENNER B, BULLER HR, DECOUSUS H, GALLUS MD, et al. Oral rivaroxaban for symptomatic venous thromboembolism. N Engl J Med. 2010;363(26):2499-510.

35. NUNNELEE JD. Review of an article: oral rivaroxaban for symptomatic venous thromboembolism. The EINSTEIN Investigators et al. N Engl J Med 2010; 363(26):2499-2510. J Vasc Nurs. 2011;29(2):89.

36. ROMUALDI E, DONADINI MP, AGENO W. Oral rivaroxaban after symptomatic venous thromboembolism: the continued treatment study (EINSTEIN-extension study). Expert review of cardiovascular therapy. 2011;9(7):841-4.

37. AGNELLI G, BULLER HR, COHEN A, CURTO M, GALLUS AS, JOHNSON M, et al. Oral apixaban for the treatment of acute venous thromboembolism. $\mathrm{N}$ Engl $\mathrm{J}$ Med. 2013;369(9):799-808.

38. AGNELLI G, BULLER HR, COHEN A, CURTO M, GALLUS AS, JOHNSON M, et al. Apixaban for extended treatment of venous thromboembolism. N Engl J Med. 2013;368(8):699-708.

39. HOKUSAI VTEI, BULLER HR, DECOUSUS H, GROSSO MA, MERCURI M, MIDDELDORP S, et al. Edoxaban versus warfarin for the treatment of symptomatic venous thromboembolism. N Engl J Med. 2013;369(15):1406-15.

40. ERIKSSON BI, DAHL OE, ROSENCHER N, KURTH AA, VAN DIJK CN, FROSTICK SP, et al. Dabigatran etexilate versus enoxaparin for prevention of venous thromboembolism after total hip replacement: a randomised, double-blind, non-inferiority trial. Lancet. 2007;370(9591):949-56.

41. ERIKSSON BI, DAHL OE, ROSENCHER N, KURTH AA, VAN DIJK CN, FROSTICK SP, et al. Oral dabigatran etexilate vs. subcutaneous enoxaparin for the prevention of venous thromboembolism after total knee replacement: the RE-MODEL randomized trial. Journal of thrombosis and haemostasis : JTH. 2007;5(11):2178-85.

42. ERIKSSON BI, BORRIS LC, FRIEDMAN RJ, HAAS S, HUISMAN MV, KAKKAR AK, et al. Rivaroxaban versus enoxaparin for thromboprophylaxis after hip arthroplasty. N Engl J Med. 2008;358(26):2765-75.

43. KAKKAR AK, BRENNER B, DAHL OE, ERIKSSON BI,
MOURET P, MUNTZ J, et al. Extended duration rivaroxaban versus short-term enoxaparin for the prevention of venous thromboembolism after total hip arthroplasty: a double-blind, randomised controlled trial. Lancet. 2008;372(9632):31-9.

44. LASSEN MR, AGENO W, BORRIS LC, LIEBERMAN JR, ROSENCHER N, BANDEL TJ, et al. Rivaroxaban versus enoxaparin for thromboprophylaxis after total knee arthroplasty. The New England journal of medicine. 2008;358(26):2776-86.

45. TURPIE AG, LASSEN MR, DAVIDSON BL, BAUER KA, GENT M, KWONG LM, et al. Rivaroxaban versus enoxaparin for thromboprophylaxis after total knee arthroplasty (RECORD4): a randomised trial. Lancet. 2009;373(9676):1673-80.

46. ALVES C, BATEL-MARQUES F, MACEDO AF. Apixaban and rivaroxaban safety after hip and knee arthroplasty: a meta-analysis. J Cardiovasc Pharmacol Ther. 2012;17(3):266-76.

47. COHEN AT, SPIRO TE, BULLER HR, HASKELL L, HU D, HULL R, et al. Extended-duration rivaroxaban thromboprophylaxis in acutely ill medical patients: MAGELLAN study protocol. J Thromb Thrombolysis. 2011;31(4):407-16.

48. GOLDHABER SZ, LEIZOROVICZ A, KAKKAR AK, HAAS SK, MERLI G, KNABB RM, et al. Apixaban versus enoxaparin for thromboprophylaxis in medically ill patients. The New England journal of medicine. 2011;365(23):2167-77.

49. COHEN AT, HARRINGTON R, GOLDHABER SZ, HULL $\mathrm{R}$, GIBSON CM, HERNANDEZ AF, et al. The design and rationale for the Acute Medically Ill Venous Thromboembolism Prevention with Extended Duration Betrixaban (APEX) study. American heart journal. 2014;167(3):335-41

50. POLLACK CV, JR., REILLY PA, EIKELBOOM J, GLUND $S$, VERHAMME P, BERNSTEIN RA, et al. Idarucizumab for Dabigatran Reversal. The New England journal of medicine. 2015;373(6):511-20.

51. CONNOLLY SJ, MILLING TJ, JR., EIKELBOOM JW, GIBSON CM, CURNUTTE JT, GOLD A, et al. Andexanet Alfa for Acute Major Bleeding Associated with Factor Xa Inhibitors. N Engl J Med. 2016;375(12):1131-41.

52. VERHEUGT FW, GRANGER CB. Oral anticoagulants for stroke prevention in atrial fibrillation: current status, special situations, and unmet needs. Lancet. 2015;386(9990):303-10. 